\title{
Effect of treadmill exercise and bone marrow stromal cell engraftment on activation of BDNF-ERK-CREB signaling pathway in the crushed sciatic nerve
}

\author{
Tae-Beom Seo, Yeong-Hyun Cho, Hyuk Sakong, Young-Pyo Kim* \\ Department of Kinesiology, College of Natural Science, Jeju National University, Jeju, Korea
}

The effect of combined approach of exercise training and bone marrow stromal cell (BMSC) engraftment on activation of brain-derived neurotrophic factor (BDNF)-extracellular signal-regulated kinase 1 and 2 (ERK1/2)-cyclic adenosine monophosphate response element-binding protein (CREB) signaling pathway after sciatic nerve injury (SNI) was investigated. Sixty male Sprague-Dawley rats divided into the normal control, nonexercise (NEX), exercise training (EX), BMSC transplantation (TP), and exercise training+BMSC transplantation (EX+TP) groups 4 weeks after SCI. Exercise training was carried out on the treadmill device at $5-10 \mathrm{~m} / \mathrm{min}$ for $20 \mathrm{~min}$ for 4 weeks. Single dose of $5 \times 10^{6}$ harvested BMSC was injected into the injury area of the injured sciatic nerve. In order to evaluate induction levels of BDNF-ERK1/2-CREB signaling molecules in the whole cell and nuclear cell lysates of the injured sciatic nerve, we applied Western blot analysis. BDNF was significantly in- creased only in EX+TP compared to NEX, EX, and TP groups. Phosphoinositide-dependent kinase-1 was more increased in EX, TP, and EX+TP groups than NEX group, but EX+TP group showed the most upregulation of phosphorylated protein kinase B compared to other groups. In addition, in the whole cell lysate, phosphorylated ERK1/2, but not activating transcription factor-3 (ATF-3) and phosphorylated CREB, was significantly increased in TP and EX+TP groups. In the nuclear cell lysate, ATF-3 and phosphorylated CREB were strongly activated in EX+TP group compared to EX group. Regular exercise training combined with BMSC engraftment would seem to be more effective in controlling activation of regeneration-related signaling pathway after SNI.

Keywords: Sciatic nerve, Bone marrow stromal cell, Treadmill exercise, Brain-derived neurotrophic factor

\section{INTRODUCTION}

Despite regenerative capacity of axonal regrowth in the injured peripheral nervous system (PNS), many patients with sciatic nerve injury (SNI) suffer from long-term disability and decrease in the quality of life (McGregor and English, 2019; Zheng et al., 2016). Also, although clinical and animal previous studies have suggested various therapeutic approaches to improve axonal regeneration after SNI for decades, it is thought that many studies on cell biological changes for functional recovery should be carried out in more detail.

Axonal regeneration in the injured sciatic nerve is closely associated with activation of a specific signaling pathway including neurotrophic factors and regulation molecules of cell cycle (Allodi et al., 2012; Hei et al., 2017). Neurotrophic factors are comprised of brain-derived neurotrophic factor (BDNF), nerve growth factor, neurotrophin-3, and neurotrophin-4/5. Among these neurotrophic factors, BDNF has been known as one of the most effective molecules for regeneration of the injured peripheral nerves (Krakowiak et al., 2015). Zheng et al. (2016) reported that BDNF induction during sciatic nerve regeneration could result in improvement of the intrinsic axonal sprouting ability through activation of cyclic adenosine 3,5-monophosphate (cAMP) and signal transducer and activator of transcription- 3 signaling pathways. The cyclic adenosine monophosphate response element-binding protein (CREB) is phosphorylated by $\mathrm{Ca}^{2+}$ dependent transcription of exon IV-con-

\footnotetext{
${ }^{*}$ Corresponding author: Young-Pyo Kim (D) https://orcid.org/0000-0001-6662-1393 Department of Kinesiology, College of Natural Science, Jeju National University, 102 Jejudaehak-ro, Jeju 63243, Korea

Email: kimyp@jejunu.ac.kr

Received: November 5, 2021 / Accepted: November 23, 2021
}

This is an Open Access article distributed under the terms of the Creative Commons Attribution Non-Commercial License (https://creativecommons.org/licenses/by-nc/4.0/) which permits unrestricted non-commercial use, distribution, and reproduction in any medium, provided the original work is properly cited. 
taining BDNF mRNA (Lonze and Ginty, 2002; West et al., 2001) and extracellular signal-regulated kinase 1 and 2 (ERK1/2) signal cascade (Song et al., 2005).

To facilitate peripheral nerve regeneration, recent studies have suggested new therapeutic technique such as transplantation of several types of cells (Jiang et al., 2017). Tremp et al. (2015) found that autologous stem cell engraftment in rat model with SNI increased the length of the regenerating axon, and Abbas et al. (2019) demonstrated that bone marrow stromal cell (BMSC) after hindlimb replantation improved sciatic functional index through increases of the number of regenerating axons and Schwann cell proliferation.

In addition to cell transplantation therapy, low-intensity physical activity is another approach that can be applied to sciatic nerve regeneration (Gómez-Pinilla et al., 2002; Seo et al., 2006). Exercise-induced functional recovery after SNI was induced by endogenous BDNF transported in both anterograde and retrograde directions (Risahl and Fainzilber, 2010) as well as the initiation of exercise training from early stage of sciatic nerve regeneration could upregulate an activity in cell cycle regulation proteins to promote Schwann cell proliferation and motor function recovery (Seo et al., 2006).

With these previous findings on regeneration, exercise training, and cell transplantation approaches are a positive regulator related to activation of a specific signaling pathway in the injured sciatic nerve. But, a clear mechanism on the combined treatment of exercise and BMSC transplantation for sciatic nerve regeneration is poor understood and insufficient. Therefore, the purpose of present study was to confirm whether exercise training in combination with BMSC transplantation might activate BDNF-ERK1/2CREB signaling pathway in the injured sciatic nerves.

\section{MATERIALS AND METHODS}

\section{Experimental animals}

Present experiment used male Sprague-Dawley rats (8 weeks old), and they were randomly classified into the normal group ( $\mathrm{n}=$ 12) and nonexercise (NEX, $n=12$ ), exercise (EX, $n=12$ ), BMSC transplantation (TP, $\mathrm{n}=12$ ), and exercise+BMSC transplantation $(\mathrm{EX}+\mathrm{TP}, \mathrm{n}=12)$ groups 4 weeks after SNI. Animals room temperature and humidity were maintained at $22^{\circ} \mathrm{C}$ and $60 \%$, respectively. They were accepted to eat commercial rat chow (Samyang Co., Seoul, Korea) and water ad libitum. This experiment obtained approval by the ethics committee of Jeju National University (approval number: 2019-0028).

\section{Sciatic nerve injury}

After anesthesia using an animal inhalation narcosis control (Jeungdo Bio \& Plant, Seoul, Korea), the left sciatic nerve was exposed and crushed with a pair of forceps held tightly for $1 \mathrm{~min}$ and $30 \mathrm{sec}$ at intervals (Seo et al., 2006). After surgery, anesthetized animals were then placed on a heating pad maintained at $37^{\circ} \mathrm{C}$, and then they were put in their cages for resting. All rats were sacrificed 3,7 , and 14 days later.

\section{BMSC culture and transplantation}

BMSCs were collected from femur and tibia of young rats (4 weeks old), as the extraction technique described by Kim et al. (2018) and Nakano et al. (2013). Cells were cultured in Dulbecco's modified Eagle's medium with $20 \%$ fetal calf serum. Five to 7 days after cell culture, BMSCs adhered to the base on the culture dishes proliferated to a density of ca. $5 \times 10^{6}$ in one dish. Single dose of $5 \times 10^{6}$ harvested BMSCs $(30-\mu \mathrm{L}$ phosphate-buffered saline) was injected into the injury area using a 30-gauge needle.

\section{Treadmill exercise}

All animals completed adaptation period for a week, and then participated in major experiment. Rats in exercise groups had rest for 2 days after injury and carried out low-intensity treadmill exercise. Walking exercise was performed at $5-10 \mathrm{~m} / \mathrm{min}$ for $20 \mathrm{~min}$ with no inclination for 4 weeks on the treadmill device (Jeungdo Bio \& Plant).

\section{Western blot analysis}

The dissected sciatic nerves were rinsed with phosphate-buffered saline, and lysed in Triton lysis buffer. Nucleus and cytoplasm were separated using by nuclear extraction buffer and cytosol extraction buffer. Denatured proteins were separated on sodium dodecyl sulphate-polyacrylamide gel and then transferred onto polyvinylidene difluoride membrane on ice at $200 \mathrm{~mA}$ for $2 \mathrm{hr}$. The membranes were blocked with $5 \%$ skim milk, $0.1 \%$ Tween 20 in tris buffered saline for $30 \mathrm{~min}$ at room temperature. Then, the membranes were incubated overnight with primary antibodies at $4^{\circ} \mathrm{C}$. Protein $(20 \mu \mathrm{g})$ was used for Western blot analysis using antiBDNF rabbit monoclonal antibody (1:1,000, Cell Signaling Biotechnology, Danvers, MA, USA), anti-phosphorylated 3-phosphoinositide-dependent protein kinase-1 (p-PDK1) rabbit polyclonal antibody (1:1,000, Cell Signaling Biotechnology), anti-phosphorylated protein kinase B (p-Akt) rabbit monoclonal antibody (1:1,000, Cell Signaling Biotechnology), anti-phosphorylated CREB rabbit monoclonal antibody (1:1,000, Cell Signaling Bio- 
technology), anti-ATF3 rabbit monoclonal antibody (1:1,000, Cell Signaling Biotechnology), anti-phosphorylated ERK1/2 (p-ERK1/2) rabbit monoclonal antibody (1:1,000, Cell Signaling Biotechnology), anti- $\beta$-actin (1:2,000, Santa Cruz Biotechnology, Santa Cruz, CA, USA) antibodies, and goat anti-mouse or goat anti-rabbit horseradish peroxidase-conjugated secondary antibody $(1: 1,000$, GeneTex Inc., Irvine, CA, USA) were used. The blotting proteins were detected by using Westar ECL substrates (Cyanagen, Bologna, Italy). Analysis of protein density was performed using Chemidoc (Bio-Rad, Hercules, CA, USA).

\section{Statistical analysis}

All the data is presented as a mean \pm standard error. Statistical analysis was performed using one-way analysis of variance followed by Duncan post hoc test. The significance level was set at $P<0.05$. All graphs were shown by using Prism 6 (GraphPad, La Jolla, CA, USA).

\section{RESULTS}

\section{Activation of BDNF-Akt signaling pathway 28 days after SNI}

To confirm the effect of combination approach of exercise train- ing and BMSC engraftment, we carried out analysis of biochemical changes using the injured sciatic nerves. As shown in Fig. 1, BDNF was significantly increased only in EX+TP compared to NEX, EX, and TP groups. Phosphorylated PDK-1, downstream molecule of BDNF, showed more upregulation in EX, TP, and $\mathrm{EX}+\mathrm{TP}$ groups, but there was no statistically significant difference among EX, TP, and EX+TP groups. Lastly, EX, TP, and EX+TP groups significantly increased phosphorylation of Akt, and EX+ TP group showed the most increase of $\mathrm{p}$-Akt expression compared to other groups.

\section{Activation of ERK1/2-CREB pathway in whole cell lysate 28 days after $\mathrm{SNI}$}

p-ERK1/2 and CREB have been known as a mediator for cell survival and neuroplasticity in the regenerating nervous system (Ishii et al., 2013). As shown in Fig. 2, in whole cell lysate, p-ERK1/2 was significantly enhanced in EX group compared to NEX group, and TP and EX+TP group showed more increase of p-ERK1/2 levels than those in EX group. in addition, activating transcription factor-3 (ATF-3) expression levels were further upregulated in $\mathrm{EX}$, but there is no difference among NEX, TP, and EX+TP groups. p-CREB levels were not significant in NEX, EX, TP, and $\mathrm{EX}+\mathrm{TP}$ groups.

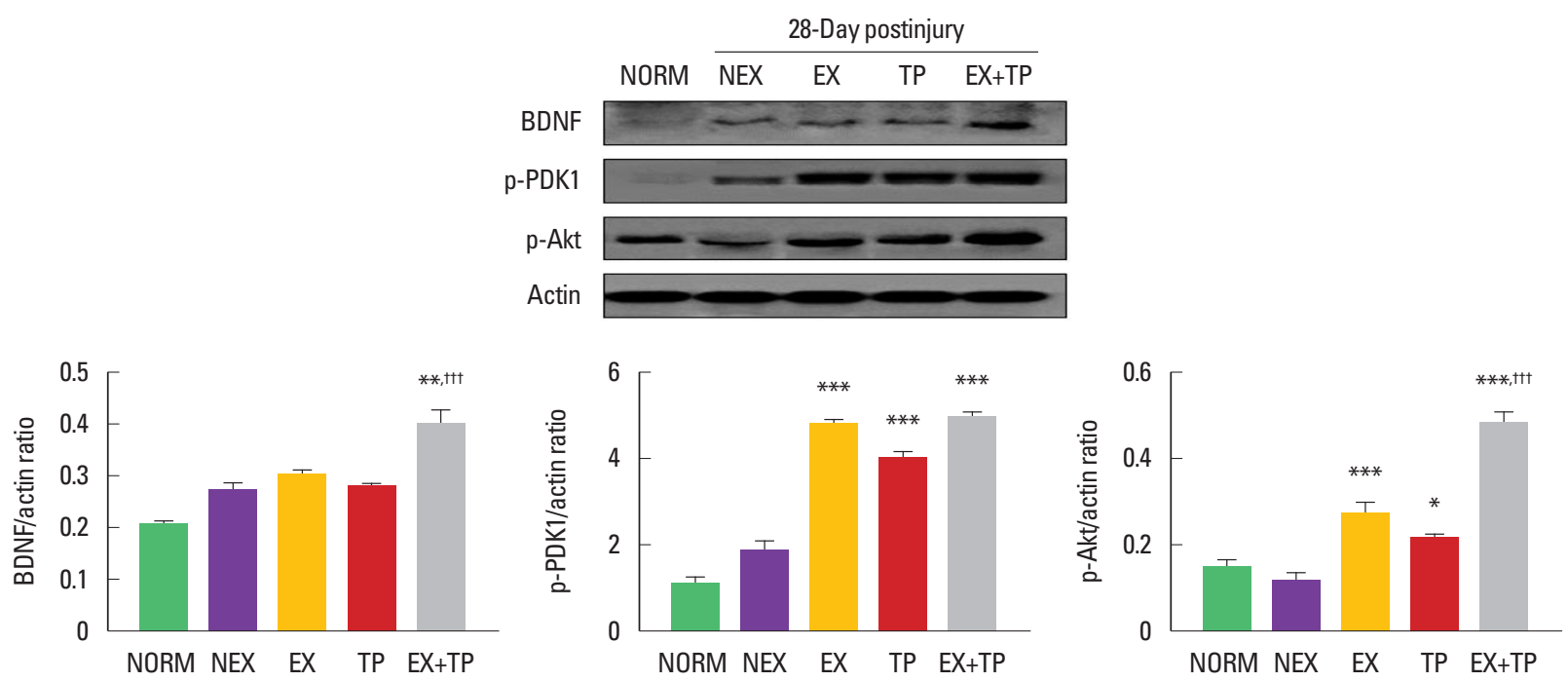

Fig. 1. Combination of exercise training and bone marrow stromal cell (BMSC) engraftment activated BDNF-Akt signaling pathway 28 days after sciatic nerve injury. Only EX+TP group strongly increased BDNF levels, but p-PDK1 was significantly enhanced in EX, TP, and EX+TP groups than those in NEX group. Additionally, EX+TP group leads to greater phosphorylation of Akt compared to EX group. Upper panel: representative expression of BDNF, phosphorylated PDK-1 (p-PDK-1), and p-Akt. Lower left panel: the density ratio of BDNF to $\beta$-actin band. Lower middle panel: the density ratio of p-PDK-1 to $\beta$-actin band. Lower right panel: the density ratio of p-Akt to $\beta$-actin band. BDNF, brain-derived neurotrophic factor; Akt, protein kinase B; PDK-1, phosphoinositide-dependent kinase-1; NORM, normal group; NEX, nonexercise training group; EX, exercise training group; TP, BMSC transplantation group; EX+TP, exercise training, and BMSC transplantation group. ${ }^{*} P<0.05$, ${ }^{* *} P<0.01$, ${ }^{* * *} P<0.001$ vs. SED group. ${ }^{\text {tt }} P<0.001$ vs. EX group. 


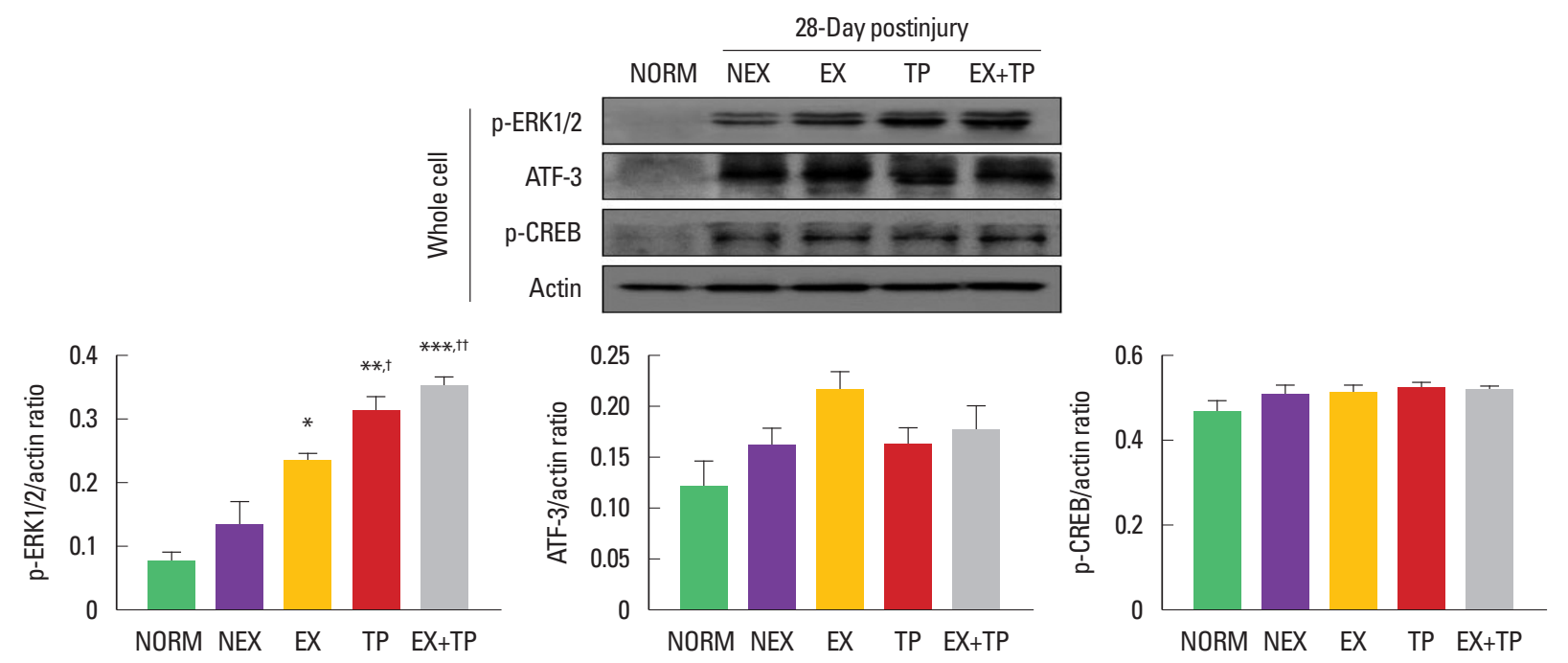

Fig. 2. Combination of exercise training and bone marrow stromal cell (BMSC) engraftment regulated ERK1/2 and CREB phosphorylation 28 days after sciatic nerve injury (SNI). In whole cell lysate, ERK1/2 phosphorylation was significantly upregulated in TP and EX+TP compared to EX group, but ATF-3 and CREB did not showed a statistically significant difference between all groups. Upper panel: representative expression of phosphorylated ERK1/2 (p-ERK1/2), ATF-3, and p-CREB. Lower left panel: the density ratio of $p$-ERK1/2 to $\beta$-actin band. Lower middle panel: the density ratio of AFT-3 to $\beta$-actin band. Lower right panel: the density ratio of $p$-CREB to $\beta$-actin band. ERK1/2, extracellular signal-regulated kinase 1 and 2; CREB, cyclic adenosine monophosphate response element-binding protein; ATF-3, activating transcription factor-3; NORM, normal group; NEX, nonexercise training group; EX, exercise training group; TP, BMSC transplantation group; EX+TP, exercise training, and BMSC transplantation group. ${ }^{*} P<0.05,{ }^{* *} P<0.01,{ }^{* *} P<0.001$ vs. SED group. ${ }^{\dagger} P<0.05,{ }^{\dagger \dagger} P<0.01$ vs. EX group.
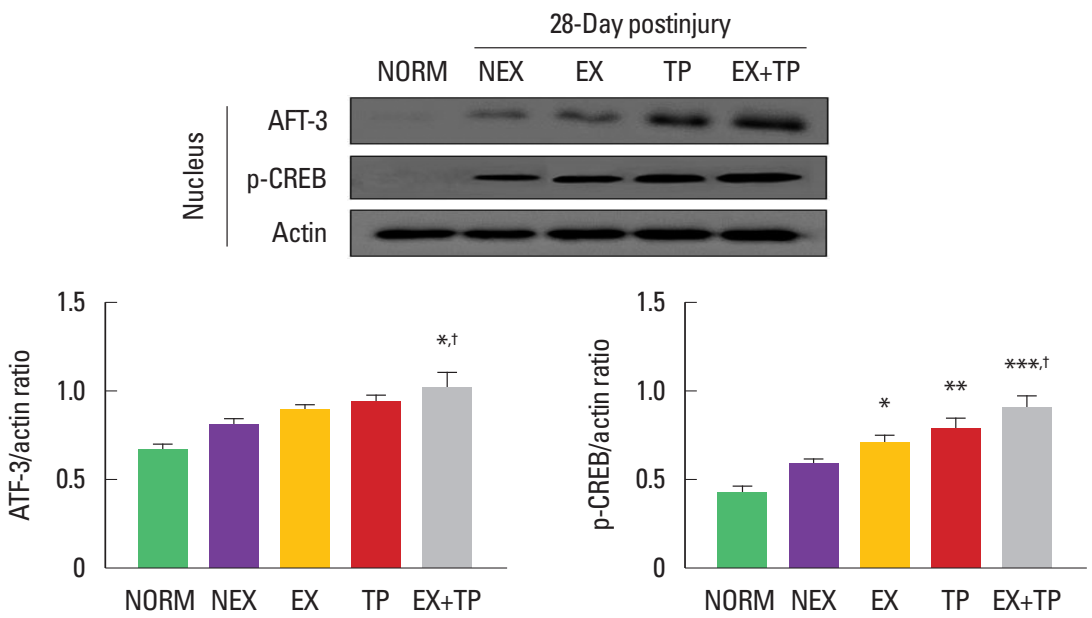

Fig. 3. Combination of exercise training and bone marrow stromal cell (BMSC) engraftment activated transcription factors in the nucleus 28 days after sciatic nerve injury (SNI). In the nuclear cell lysate, ATF-3 showed a significant difference only in EX+TP group. phosphorylation of CREB factor was upregulated in EX, TP, and EX+ TP groups, and EX+TP group led to a greater increase in phosphorylation of CREB than those in EX group. Upper panel: representative expression of ATF-3 and phosphorylated CREB (p-CREP) in the nuclear cell lysate. Lower left panel: the density ratio of ATF-3 to $\beta$-actin band. Lower right panel: the density ratio of $p$-CREB to $\beta$-actin band. ATF-3, activating transcription factor-3; CREB, cyclic adenosine monophosphate response element-binding protein; NORM, normal group; NEX, nonexercise training group; EX, exercise training group; TP, BMSC transplantation group; EX+TP, exercise training, and BMSC transplantation group. ${ }^{*} P<0.05$, ${ }^{* *} P<0.01$, ${ }^{* * *} P<0.001$ vs. SED group. ${ }^{\dagger} P<0.05$ vs. EX group.

\section{Activation of ATF-3 and p-CREB in the nucleus 28 days after SNI}

To demonstrate translocation of ATF-3 and CREB from the cytosol to the nucleus of Schwann cells after SNI, we analyzed expres- sion levels of ATF-3 and CREB into the nuclear cell lysate of Schwann cells. As shown in Fig. 3, in the nuclear cell lysate, inductions of ATF-3 were significantly enhanced only in EX+TP groups compared to other groups. CREB phosphorylation was dramatically 
increased in EX, TP, and EX+TP groups, and only EX+TP group led to the greater increase in $\mathrm{p}-\mathrm{CREB}$ than those in EX group.

\section{DISCUSSION}

Unlike regeneration characteristics of central nervous system, it is known that PNS can spontaneously overcome the histological and functional problems induced by nerve injury (Huebner and Strittmatter, 2009). But, such voluntary regeneration has limitations in showing various results depending on the therapeutic methods and environment. Therefore, this study tried to find out cellular mechanisms involved in sciatic nerve regeneration through applying exercise training and BMSC transplantation.

BDNF is a good candidate for remarkably increasing axonal lengthening and Schwann cell proliferation from the initial phase of regeneration (Ma et al., 2011), and it contributes to regulating nutrition supports for axonal sprouting in the distal region to the injury site (Zheng et al., 2016). Additionally, BDNF serves as a regulator promoting various downstream molecules, including cAMP and ERK1/2, to make regenerative environment in the injured PNS (Jiang et al., 2017). Thus, we examined BDNF induction levels in the injured sciatic nerve, and EX+TP group dramatically increased BDNF compared to NEX group, but not in EX and TP groups. Many previous studies on the role of BDNF in peripheral nerve regeneration have reported that $\mathrm{BDNF}$ is induced by a two-fold increase from 3 days to 11 days after SNI (BałkowiecIskra et al., 2011; Senger et al., 2018), as well as applying exercise training (Gómez-Pinilla et al., 2002) and BMSC transplantation technique (Kemp et al., 2008) further accelerates axonal lengthening via activation of BDNF in Schwann cells of the injured sciatic nerves. The contradictory finding between previous studies (result within 2 weeks postinjury) and present data (result at 4 weeks postinjury) are due to the different time point of BDNF analysis. However, it is thought that in combination approaches of exercise training and BMSC transplantation possessed a synergistic effect with the greatest increase of BDNF at 4 weeks later.

PDK1 and Akt are downstream proteins regulated by BDNF, which play an essential role in cell survival, neurogenesis, and axon growth in the injured nervous system (Vanhaesebroeck and Alessi, 2000). In present study, phosphorylated PDK1 was significantly upregulated in EX, TP, and EX+TP groups compared to NEX group, and phosphorylated Akt showed the greatest enhancement in EX+TP group. These findings indicated that PDK1 and Akt molecules have a positive potential for sciatic nerve regeneration and stimulated by exercise training and BMSC engrafting. How- ever, unlike our findings on PDK1-related regeneration, previous study reported by Kim et al. (2021) suggested promoting axonal regeneration by the chemical inhibition of PDK1 in vivo and in vitro. Although the role of PDK1 in peripheral nerve regeneration is somewhat controversial, exercise training and BMSC engrafting may be a regulator to activate PDK1 and Akt signaling pathway that promote regeneration.

In addition to BDNF-Akt pathway, ERK1/2 protein, one molecule of the mitogen-activated protein kinase, is another key molecule in development and regeneration in PNS, and it is usually expressed in Schwann cell of the injured sciatic nerve for enhancing remyelination (Ishii et al., 2013). Seo et al. (2009) suggested that phosphorylated ERK1/2 protein was induced in Schwann cells and dorsal root ganglion sensory neurons from the early phase of sciatic nerve regeneration, and treadmill training strongly activated p-ERK1/2 for proliferation of Schwann cells and neurite outgrowth of dorsal root ganglion sensory neurons. Additionally, p-ERK1/2 translocated into the nucleus activates CREB at serine residue 133 , improving cell survival and neuropathic pain after central and peripheral nerve injury (Lu and Malemud, 2019). Present study confirmed that in whole cell lysate, p-ERK1/2 was significantly increased in TP and EX+TP groups as well as significant differences in expression levels of ATF-3 and p-CREB appeared only in the nuclear cell lysate, but not whole cell lysate. In previous studies, Miyabe and Miletic (2005) reported that p-ERK1/2 and p-CREB were detected from $2 \mathrm{hr}$ after SNI and application of ERK1/2 inhibitor downregulated sciatic nerve ligation-associated CREB phosphorylation, and Patodia and Raivich (2012) suggested that various transcription factors including CREB and ATF3 were activated in the nucleus during nerve regeneration, and they regulated expression of target genes and the regenerative response of the injured neuron. The results of these previous studies support present findings that activations of ATF- 3 and CREB in the nucleus by the application of exercise training and BMSC engraftment have a positive potential for sciatic nerve regeneration.

Given these findings reported in present study, regular exercise training combined with BMSC engraftment seem to be more effective in controlling activation of regeneration-related signaling pathway after SNI.

\section{CONFLICT OF INTEREST}

No potential conflict of interest relevant to this article was reported. 


\section{ACKNOWLEDGMENTS}

This work was supported by the National Research Foundation of Korea (NRF) grant funded by the Korea government (MSIT) (No. 2019R1F1A1063706). This research was supported by the 2020 scientific promotion program funded by Jeju National University.

\section{REFERENCES}

Abbas OL, Özatik O, Gönen ZB, Koçman AE, Dağ I, Özatik FY, Bahar D, Musmul A. Bone marrow mesenchymal stem cell transplantation enhances nerve regeneration in a rat model of hindlimb replantation. Plast Reconstr Surg 2019;143:758e-768e.

Allodi I, Udina E, Navarro X. Specificity of peripheral nerve regeneration: interactions at the axon level. Prog Neurobiol 2012;98:16-37.

Bałkowiec-Iskra E, Vermehren-Schmaedick A, Balkowiec A. Tumor necrosis factor- $\alpha$ increases brain-derived neurotrophic factor expression in trigeminal ganglion neurons in an activity-dependent manner. Neuroscience 2011;180:322-333.

Gómez-Pinilla F, Ying Z, Roy RR, Molteni R, Edgerton VR. Voluntary exercise induces a BDNF-mediated mechanism that promotes neuroplasticity. J Neurophysiol 2002;88:2187-2195.

Hei WH, Almansoori AA, Sung MA, Ju KW, Seo N, Lee SH, Kim BJ, Kim $\mathrm{SM}$, Jahng JW, He $\mathrm{H}$, Lee JH. Adenovirus vector-mediated ex vivo gene transfer of brain-derived neurotrophic factor (BDNF) tohuman umbilical cord blood-derived mesenchymal stem cells (UCB-MSCs) promotescrush-injured rat sciatic nerve regeneration. Neurosci Lett 2017;643:111-120.

Huebner EA, Strittmatter SM. Axon regeneration in the peripheral and central nervous systems. Results Probl Cell Differ 2009;48:339-351.

Ishii A, Furusho M, Bansal R. Sustained activation of ERK1/2 MAPK in oligodendrocytes and schwann cells enhances myelin growth and stimulates oligodendrocyte progenitor expansion. J Neurosci 2013;33: 175-186.

Jiang L, Jones S, Jia X. Stem cell transplantation for peripheral nerve regeneration: current options and opportunities. Int J Mol Sci 2017;18:94.

Kemp SW, Walsh SK, Midha R. Growth factor and stem cell enhanced conduits in peripheral nerve regeneration and repair. Neurol Res 2008; 30:1030-1038

Kim H, Lee J, Cho Y. PDK1 is a negative regulator of axon regeneration. Mol Brain 2021;14:31.

Kim SH, Cho JH, Lee YH, Lee JH, Kim SS, Kim MY, Lee MG, Kang WY, Lee KS, Ahn YK, Jeong MH, Kim HS. Improvement in left ventricular function with intracoronary mesenchymal stem cell therapy in a pa- tient with anterior wall ST-segment elevation myocardial infarction. Cardiovasc Drugs Ther 2018;32:329-338.

Krakowiak J, Liu C, Papudesu C, Ward PJ, Wilhelm JC, English AW. Neuronal BDNF signaling is necessary for the effects of treadmill exercise on synaptic stripping of axotomized motoneurons. Neural Plast 2015; 2015:392591.

Lonze BE, Ginty DD. Function and regulation of CREB family transcription factors in the nervous system. Neuron 2002;35:605-623.

Lu N, Malemud CJ. Extracellular signal-regulated kinase: a regulator of cell growth, inflammation, chondrocyte and bone cell receptor-mediated gene expression. Int J Mol Sci 2019;20:3792.

Ma Z, Wang J, Song F, Loeb JA. Critical period of axoglial signaling between neuregulin-1 and brain-derived neurotrophic factor required for early Schwann cell survival and differentiation. J Neurosci 2011;31: 9630-9640

McGregor CE, English AW. The role of BDNF in peripheral nerve regeneration: activity-dependent treatments and Val66Met. Front Cell Neurosci 2019;12:522.

Miyabe T, Miletic V. Multiple kinase pathways mediate the early sciatic ligation-associated activation of CREB in the rat spinal dorsal horn. Neurosci Lett 2005;381:80-85.

Nakano N, Nakai Y, Seo TB, Homma T, Yamada Y, Ohta M, Suzuki Y, Nakatani T, Fukushima M, Hayashibe M, Ide C. Effects of bone marrow stromal cell transplantation through CSF on the subacute and chronic spinal cord injury in rats. PLoS One 2013;8:e73494.

Patodia S, Raivich G. Role of transcription factors in peripheral nerve regeneration. Front Mol Neurosci 2012;5:8.

Rishal I, Fainzilber M. Retrograde signaling in axonal regeneration. Exp Neurol 2010;223:5-10.

Senger JB, Verge VMK, Chan KM, Webber CA. The nerve conditioning lesion: a strategy to enhance nerve regeneration. Ann Neurol 2018;83: 691-702.

Seo TB, Han IS, Yoon JH, Hong KE, Yoon SJ, Namgung U. Involvement of $\mathrm{Cdc} 2$ in axonal regeneration enhanced by exercise training in rats. Med Sci Sports Exerc 2006;38:1267-1276.

Seo TB, Oh MJ, You BG, Kwon KB, Chang IA, Yoon JH, Lee CY, Namgung $\mathrm{U}$. ERK1/2-mediated Schwann cell proliferation in the regenerating sciatic nerve by treadmill training. J Neurotrauma 2009;26:1733-1744.

Song XS, Cao JL, Xu YB, He JH, Zhang LC, Zeng YM. Activation of ERK/ CREB pathway in spinal cord contributes to chronic constrictive injuryinduced neuropathic pain in rats. Acta Pharmacol Sin 2005;26:789-798.

Tremp M, Meyer Zu Schwabedissen M, Kappos EA, Engels PE, Fischmann A, Scherberich A, Schaefer DJ, Kalbermatten DF. The regeneration potential after human and autologous stem cell transplantation in a rat sciatic nerve injury model can be monitored by MRI. Cell Transplant 
2015;24:203-211.

Vanhaesebroeck B, Alessi DR. The PI3K-PDK1 connection: more than just a road to PKB. Biochem J 2000;346 Pt 3:561-576.

West AE, Chen WG, Dalva MB, Dolmetsch RE, Kornhauser JM, Shaywitz AJ, Takasu MA, Tao X, Greenberg ME. Calcium regulation of neuro- nal gene expression. Proc Natl Acad Sci U S A 2001;98:11024-11031.

Zheng J, Sun J, Lu X, Zhao P, Li K, Li L. BDNF promotes the axonal regrowth after sciatic nerve crush through intrinsic neuronal capability upregulation and distal portion protection. Neurosci Lett 2016;621:1-8. 